\title{
Correction to: Reldesemtiv in Patients with Spinal Muscular Atrophy: a Phase 2 Hypothesis-Generating Study
}

\author{
Stacy A. Rudnicki ${ }^{1}$. Jinsy A. Andrews ${ }^{1,2} \cdot$ Tina Duong $^{3} \cdot$ Bettina M. Cockroft ${ }^{1,4} \cdot$ Fady I. Malik $^{1} \cdot$ Lisa Meng $^{1}$. \\ Jenny Wei ${ }^{1}$. Andrew A. Wolff ${ }^{1}$. Angela Genge ${ }^{5}$. Nicholas E. Johnson ${ }^{6,7}$. Carolina Tesi-Rocha ${ }^{3}$. Anne M. Connolly ${ }^{8,9}$. \\ Basil T. Darras ${ }^{10}$. Kevin Felice ${ }^{11}$. Richard S. Finkel ${ }^{12,13}$. Perry B. Shieh ${ }^{14}$. Jean K. Mah ${ }^{15}$. Jeffrey Statland ${ }^{16}$. \\ Craig Campbell $^{17}$. Ali A. Habib ${ }^{18}$. Nancy L. Kuntz ${ }^{19} \cdot$ Maryam Oskoui $^{20} \cdot$ John W. Day $^{3}$
}

Published online: 1 November 2021

(c) The American Society for Experimental NeuroTherapeutics, Inc. 2021

\section{Correction to: Neurotherapeutics} https://doi.org/10.1007/s13311-020-01004-3

This article has been updated to add the author Richard S. Finkel.

The original article has been corrected.

Publisher's Note Springer Nature remains neutral with regard to jurisdictional claims in published maps and institutional affiliations.

The original article can be found online at https://doi.org/10.1007/ s13311-020-1004-3.

\section{John W. Day}

jwday@stanford.edu

Cytokinetics, Incorporated, South San Francisco, CA, USA

2 Columbia University, New York, NY, USA

3 Stanford University, Stanford, CA, USA

4 Sangamo Therapeutics, Brisbane, CA, USA

5 Montreal Neurological Institute, Montreal, QC, Canada

6 Virginia Commonwealth University, Richmond, VA, USA

7 University of Utah, Salt Lake City, UT, USA

8 Nationwide Children's Hospital, Columbus, OH, USA

9 Washington University, St Louis, MO, USA

10 Boston Children's Hospital and Harvard Medical School, Boston, MA, USA

11 Hospital for Special Care, New Britain, CT, USA
12 Nemours Children's Hospital, Orlando, FL, USA

13 St. Jude Children's Research Hospital, Memphis, TN, USA

14 University of California, Los Angeles, Los Angeles, CA, USA

15 University of Calgary, Alberta Children's Hospital, Calgary, $\mathrm{AB}$, Canada

16 University of Kansas, Lawrence, KS, USA

17 Department of Pediatrics, Epidemiology and Clinical Neurological Sciences, University of Western Ontario, London Health Sciences Centre, London, ON, Canada

18 University of California, Irvine, Orange, CA, USA

19 Ann \& Robert H. Lurie Children's Hospital of Chicago, Chicago, IL, USA

20 McGill University Health Centre Research Institute, Montreal, QC, Canada 